IgG4-RD were approved by ACR and EULAR in 2019. Whether this new criteria improve the diagnosis efficiency needs to be validated in clinical practice.

Objectives: To applicate the 2019 ACR/EULAR classification criteria for the diagnosis of IgG4-RD in previously suspected patients and explore the clinical characteristics of patients with IgG4-RD according to the new classification criteria. Methods: Patients suspected of having IgG4-RD due to elevated serum IgG4 and swelling or masses in single or multiple organs were recruited in Sun Yat-Sen Memorial Hospital, Sun Yat-Sen University from May 2013 and November 2019. Demographic and clinical data were collected. The diagnosis was reevaluated with the 2011 comprehensive diagnostic criteria (CDC) for IgG4-RD and the 2019 ACR/EULAR classification criteria for lgG4-RD, respectively.

Results:

(1) There were 68 patients recruited and $59(86.8 \%)$ of them had elevated serum IgG4 ( $\geq 135 \mathrm{mg} / \mathrm{dl})$ and $53(77.9 \%)$ patients showed swelling or masses in single or multiple organs. Most patients first visit general surgery (17.6\%), gastroenterology $(16.2 \%)$, respiratory medicine $(16.2 \%)$ and rheumatology $(14.7 \%)$.

(2) According to the 2011 CDC for IgG4-RD, 4(5.9\%) patients were definite IgG4-RD, 1(1.5\%) was probable and $42(61.8 \%)$ were possible. According to the 2019 ACR/EULAR criteria, 20(29.4\%) patients were diagnosed as IgG4-RD, including the 4 definite patients using the 2011 CDC.

(3) Among the $20 \mathrm{lgG} 4-\mathrm{RD}$ patients according to the 2019 ACR/EULAR criteria, $19(95.0 \%)$ were male and median age of symptom onset was 62(46 69) years. There were $6(30.0 \%)$ patients diagnosed at rheumatology, $5(25.0 \%)$ at gastroenterology, $3(15.0 \%)$ at general surgery, $2(10.0 \%)$ at respiratory medicine and $1(5.0 \%)$ at stomatology, endocrinology, orthopedics and urinary surgery, respectively. There were $9(45.0 \%)$ patients with bilateral lacrimal or salivary glands involved, $9(45.0 \%)$ with pancreas and biliary tree involved, $5(25.0 \%)$ with retroperitoneum involved, $2(10.0 \%)$ with kidney involved and $1(5.0 \%)$ with chest involved.

(4) The median serum IgG4 of the 20 IgG4-RD patients was $15.40(4.14 \sim 55.10)$ $\mathrm{g} / \mathrm{L}$, median serum $\mathrm{IgG}$ was $27.9(17.2 \sim 50.2) \mathrm{g} / \mathrm{L}$. There were $20.0 \%(4 / 20)$ patients had elevated serum eosinophil and $93.3 \%(14 / 15)$ had elevated serum IgE. There were $60.0 \%(9 / 15)$ patients had elevated C-reactive protein, $85.7 \%(12 / 14)$ had elevated erythrocyte sedimentation rate and $26.7 \%(4 / 15)$ had hypocomplementemia. There were $35.7 \%(5 / 14)$ patients had positive rheumatoid factor and $14.3 \%(2 / 14)$ had positive antinuclear antibodies. There were $6(30.0 \%)$ patients didn't received biopsies, $8(40.0 \%)$ patients received surgical removal of salivary glands, lacrimal glands or pancreatic masses and $6(30.0 \%)$ patients received needle biopsies of the salivary glands or biopsies by bronchoscopy, gastroscopy or enteroscopy.

(5) Among the patients didn't fulfill the 2011 CDC and the 2019 ACR/EULAR criteria, there were 40 patients had elevated serum IgG4, whose serum IgG4 level were significant lower than those of IgG4-RD patients [3.66(2.39 7.68) $\mathrm{g} / \mathrm{L}$ vs. $15.40(4.14 \sim 55.10) \mathrm{g} / \mathrm{L}, P<0.001]$, and percentage of serum IgG4 $\geq 5 \times$ upper limit of normal were also lower than those of IgG4-RD patients $(27.5 \%$ vs. $73.7 \%, P=0.002)$.

Conclusion: The 2019 ACR/EULAR criteria can help diagnosing patients with IgG4-RD even lacking the tissue confirmation. Patients with moderately elevated serum IgG4 need more clinical evidence to diagnose IgG4-RD and exclude mimics.

References: This work was supported by Guangdong Medical Scientific Research Foundation (grant no. A2017093).

Disclosure of Interests: None declared

DOI: 10.1136/annrheumdis-2020-eular.4426

\section{AB1021 CHARACTERISTICS AND MANAGEMENT OF RHEUMATIC MANIFESTATION UNDER ESTROGEN RECEPTOR-TARGETING CANCER THERAPIES, DATA FROM A PROSPECTIVE REGISTRY}

A. Dr. Patroi ${ }^{1}$, L. Diekmann ${ }^{2}$, H. M. Lorenz ${ }^{3}$, B. Kraemer ${ }^{1}$, K. Benesova ${ }^{2}$, J. Leipe ${ }^{1}$. 1 University Hospital Mannheim, Division of Rheumatology, Department of Medicine V., Mannheim, Germany; ${ }^{2}$ University Hospital Heidelberg, Heidelberg, Germany, Department of Medicine V, Hematology, Oncology and Rheumatology, Heidelberg, Germany; ${ }^{2}$ University Hospital Heidelberg, Heidelberg, Germany, Department of Medicine V, Hematology, Oncology and Rheumatology, Heidelberg, Germany

Background: The knowledge about interdependencies between rheumatic manifestations and malignancies is limited. Further, reliable data on the occurrence of rheumatic symptoms as side effects of specific cancer therapies beyond checkpointinhibitor-induced immune-related adverse events are sparse. In this regard, although arthralgia under estrogen receptor-targeting therapies (aromatase inhibitors and the estrogen receptor modulator tamoxifen) has been frequently reported in oncological clinical trials and case reports, prospective data including an assessment of rheumatic manifestations by rheumatologists are lacking.

Objectives: To contribute to a better understanding of interdependencies between rheumatic manifestations and cancer/ estrogen blockade and potentially improve the management of both entities, pilot data were analysed.

Methods: Data on characteristics and treatment of rheumatic manifestations and cancer as well as their timely association were systematically, prospectively collected and analysed in the MalheuR ('malignancy and rheumatic disease') registry, a long-term, observational study designed to study patients suffering from concomitant rheumatic disease and malignancy and/or premalignant lesions.

Results: We identified 11 patients with rheumatic manifestations under estrogen receptor-targeting therapies ( 3 anastrozol, 4 letrozol, 8 tamoxifen) as part of breast cancer treatment. In addition to breast cancer one patient had a lymphoma 3 years after and another patient had a non-small cell lung cancer 2 years before breast cancer diagnosis. The patients had different cancer stages ( 5 IA, 3 IIA, 1 IIB, and 1 IVA). Their mean age at cancer diagnosis was $60.4 \pm 11.6$ years and all patients are females. The time interval between diagnosis of cancer and onset of systemic/ rheumatic symptoms was $49.5 \pm 34.0$ months. Of interest, the time interval between onset of rheumatic symptoms and first assessment by a rheumatologist was $16.9 \pm 22.3$ months. The following systemic and rheu matic symptoms were reported: arthralgia in 10, arthritis in 8 (small joints in 5 , large joints in 3 affected), morning stiffness (>30 min) in 7, IBP in 1, myalgia in 7 , sicca symptoms in 2, fever in 1 (new-onset FMF with heterozygous M694U mutation), class IV glomerulonephritis and polyserositis in 1 (with new-onset SLE) patient(s). Disease burden at baseline was rather high with a mean VAS pain of $65( \pm 12.9) / 100$. Laboratory analyses revealed an increased CRP in 6/11 $(55 \%)$ with a mean of $10.3 \pm 8.2 \mathrm{mg} / \mathrm{l}(<5)$. Autoantibody positivity was observed for ANAs in 5/10 (50\%, titers ranging from 1:80 to $1: 160)$, anti-dsDNA in 1, rheumatoid factor in only $1 / 10(10 \%)$ patients, none was anti-CCP positive. Before consulting a rheumatologist, patients were treated with NSAR 3/11 (27\%), 10/11 systemic glucocorticoids (91\%) with an initial dose of $17.5 \pm 19.5 \mathrm{mg}$ and intra-articular glucocorticoids $1 / 11(9 \%)$. Rheumatological assessment lead to initiation of csDMARDs (3/11 MTX, 1/11 SSZ, 1/11 HCQ, 1/11 AZA (later MMF/ rituximab in the SLE patient) $1 / 11$ colchicine) as corticosteroid-sparing agents with good response in the majority of patients.

Conclusion: Our data demonstrate heterogeneous rheumatic manifestations, partially with severe manifestations beyond arthralgia, so far not reported by oncological studies including follow-up, which might suggest an underreporting. Furthermore, despite close monitoring in tumor aftercare, our data show a considerable delay in referral to a rheumatologist and initiation of suitable treatment. The prospective design of the MalheuR registry enables future validation of our pilot data.

Disclosure of Interests: Alina Dr. Patroi Consultant of: Advisory board Novartis, Leonore Diekmann: None declared, Hanns-Martin Lorenz Grant/research support from: Consultancy and/or speaker fees and/or travel reimbursements: Abbvie, MSD, BMS, Pfizer, Celgene, Medac, GSK, Roche, Chugai, Novartis, UCB Janssen-Cilag, Astra-Zeneca, Lilly. Scientific support and/or educational seminars and/or clinical studies: Abbvie, MSD, BMS, Pfizer, Celgene, Medac, GSK, Roche, Chugai, Novartis, UCB, Janssen-Cilag, Astra-Zeneca, Lilly, Baxter, SOBI, Biogen, Actelion, Bayer Vital, Shire, Octapharm, Sanofi, Hexal, Mundipharm, Thermo Fisher., Consultant of: see above, Bernhard. Kraemer: None declared, Karolina Benesova Grant/research support from: Study grants for SCREENED study by Abbvie, Novartis and Rheumaliga Baden-Württemberg, Consultant of: One-time participation in Novartis advisory board., Jan Leipe Grant/research support from Consultancy and speaker fees: Abbvie, AstraZeneca, BMS, Celgene, Hospira Janssen-Cilag, LEO Pharma, Lilly, MSD, Novartis, Pfizer, Roche, Sanofi, UCB. Scientific support: Novartis, Pfizer., Consultant of: Consultancy and speaker fees: Abbvie, AstraZeneca, BMS, Celgene, Hospira, Janssen-Cilag, LEO Pharma, Lilly, MSD, Novartis, Pfizer, Roche, Sanofi, UCB. Scientific support: Novartis, Pfizer., Speakers bureau: Abbvie, AstraZeneca, BMS, Celgene, Hospira, Janssen-Cilag, LEO Pharma, Lilly, MSD, Novartis, Pfizer, Roche, Sanofi, UCB

DOI: 10.1136/annrheumdis-2020-eular.6431

\section{AB1022 1 PANNICULITIS IN RHEUMATOLOGICAL PRACTICE: THE RESULTS OF LONG-TERM FOLLOW-UP}

O. Egorova', B. Belov'. 'V.A. Nasonova Research Institute of Rheumatology, Moscow, Russian Federation

Background: Panniculitis is a group of heterogeneous inflammatory diseases that occur with damage to the subcutaneous fat (SCF), musculoskeletal system, and often internal organs

Objectives: to evaluate the course and outcome in patients with panniculitis Methods: the course of the disease was monitored in 193 patients treated at the V.A. Nasonova Research Institute of Rheumatology for 3-5 years (142 women, 51 men) aged 19 to 77 with the referral diagnosis of erythema nodosum (EN) and 
undifferentiated panniculitis and with the disease duration of 1 week to 13 years In addition to general clinical study, serum concentrations of a-1 antitrypsin, amylase, lipase, ferritin, creatinephosphokinase were determined, computed tomography of the chest organs, immunological, ultrasound scanning of the skin and SCF of the node area, tuberculosis tests and pathomorphological study of skin biopsy from the node area were performed.

Results: : as a result of laboratory and instrumental examination, the following diagnoses were made: EN associated with infection (72 people - group 1), lipodermatosclerosis (LDS) (40 -group 2), idiopathic lobular panniculitis (ILP) (32 - group 3), Löfgren's syndrome (SL) (49-group 4). During the follow-up period, 6 deaths occurred (3.1\%): in a patient with LDS due to acute heart failure and in 5 patients with ILP due to the activity of the disease which led to the development of cardiopulmonary pathology and disseminated intravascular coagulation (DIC) syndrome. In group 1, during the observation period, UE recurred in 18 people $(25 \%)$, the probable causes were: hypothermia (6), exacerbation of chronic tonsillitis (6), acute respiratory viral infections (ARVI) (4), stress (1), a rapid decrease in the dose of glucocorticoids (GC) (1), the cause is unknown (2). In group 2, recurrence occurred in 19 patients $(47.5 \%)$ due to the lack of anti-inflammatory therapy (plaquenil) (10), trauma (4), stress (3), and weight lifting (2). There was no recurrence in 7 patients in this group, however, nodes would not disappear completely, which is obviously due to the absence of plaquenil. In group 3, recurrence was registered in 24 cases $(75 \%)$ and it was associated with insufficient effect of the ongoing anti-inflammatory therapy (9), a decrease in blood glucose to minimal doses (6), hypothermia (6) and the absence/cancellation of anti-inflammatory therapy (3). In group 4, recurrence of nodes was registered in 14 cases (28.5\%), possible causes: cancellation of GC (9) and cooling/ARVI(5). Conclusion: in the observed group of patients with panniculitis mortality was $3.1 \%$. The main causes of recurrence in EN were viral-bacterial infections, and in case of ILP, LDS and SL it was insufficient effect/absence of anti-inflammatory therapy. Disclosure of Interests: None declared

DOI: 10.1136/annrheumdis-2020-eular.1433

\section{AB1023 1 CARDIAC MRI IN HYPERFERRITINAEMIC DISEASE STATES REVEALS MYOCARDIAL INFLAMMATION NOT IDENTIFIED BY ECHOCARDIOGRAPHY}

S. El-Ghazali ${ }^{1}$, H. Wilson-Morkeh ${ }^{1}$, A. Porter ${ }^{1}$, S. Thapa ${ }^{1}$, J. Mason ${ }^{1,2}$ M. Fontana ${ }^{3}$, A. Singh ${ }^{4}$, G. Cole ${ }^{2}$, T. Youngstein ${ }^{1,2}$. ${ }^{1}$ Hammersmith Hospital, Rheumatology, London, United Kingdom; ${ }^{2}$ National Heart and Lung Institute, Imperial College London, London, United Kingdom; ${ }^{3}$ Royal Free Hospital, National Amyloidosis Centre, London, United Kingdom; ${ }^{4}$ Royal Free Hospital, Rheumatology, London, United Kingdom

Background: Acutely unwell adult patients with hyperinflammatory hyperferritinaemic states are typically challenging to diagnose. Case series suggest that cardiac involvement may be common (up to $20 \%$ ) but the phenotype has not been well characterised ${ }^{1}$

The elevation of cardiac biomarkers suggests cardiac involvement, but are non-specific in acute illness. Cardiac MRI (CMR) offers the ability to characterise the myocardium and identify inflammation, and modern motion-corrected sequences now allow the assessment of patients who may struggle to breathhold in the recovery from acute illness.

Objectives: We report 3 patients who underwent CMR in the acute phase of illness with raised cardiac biomarkers.

Methods: Case records of acutely ill patients with hyperferritinaemia from two major London centres were reviewed and cases who had undergone CMR in the acute phase of illness were identified.

Results: 3 cases were identified from a cohort of 22, we report CMR findings from differing aetiologies of hyperferritinaemic states:

Case 1: A female in her 60s presented acutely unwell with fever, swollen joints and salmon pink rash. Ferritin was raised at 50574ug/L (20-300ug/L), troponin I 384ng/L (<34ng/L) and Brain Natriuretic Peptide (BNP) 324ng/L (<159ng/L). Echocardiography was normal. However CMR with T2 mapping revealed several small areas of raised signal consistent with myocardial inflammation. A diagnosis of systemic Adult Onset Stills Disease (AOSD) was made. She received IV methylprednisolone and anakinra with normalisation of cardiac biomarkers.

Case 2: A male in his 20s with known SLE with associated end stage renal failure requiring transplant. He had a previous prolonged admission secondary to $\mathrm{HLH}$. He presented with chest pain and concave shaped ST elevation on ECG. Troponin peak 2168ng/L, BNP 1334ng/L. Peak ferritin 1300ug/l.

He was initiated on colchicine for likely pericarditis. Echocardiography showed a dilated left ventricle and mildly increased wall thickness, but overall systolic function within normal limits.

CMR reported nodular patchy late gadolinium enhancement in the mid inferoseptum and inferior wall associated with areas of raised T2 mapping values. NM cardiac rest gated PET reported abnormal FDG uptake to the myocardium with sites including the apical inferior wall, apical RV insertion point and basal septal/ anterior right ventricular walls. Features were deemed in keeping with active myocarditis.

He responded to colchicine with improved troponin, and was discharged with close follow up.

Case 3: A male in his 20s presented with septic shock attributed to meningococcal septicaemia requiring ITU admission. Troponin was elevated at $>9000 \mathrm{ng} / \mathrm{L}$ Bloods demonstrated raised ferritin and features consistent with HLH were identified.

CMR reported elevated native myocardial T1/T2 signal of the lateral and mid-anterior walls in keeping with myocardial oedema. Pericardium adjacent to the anterolateral wall had elevated T1/T2 signal with hyperenhancement on delayed enhancement imaging. Tissue characterisation was in keeping with an acute myopericarditis process.

In addition to broad spectrum antibiotics to treat his underlying infection, he received therapy for $\mathrm{HLH}$ including methylprednisolone, anakinra and IVIG. He subsequently made a good recovery to treatment.

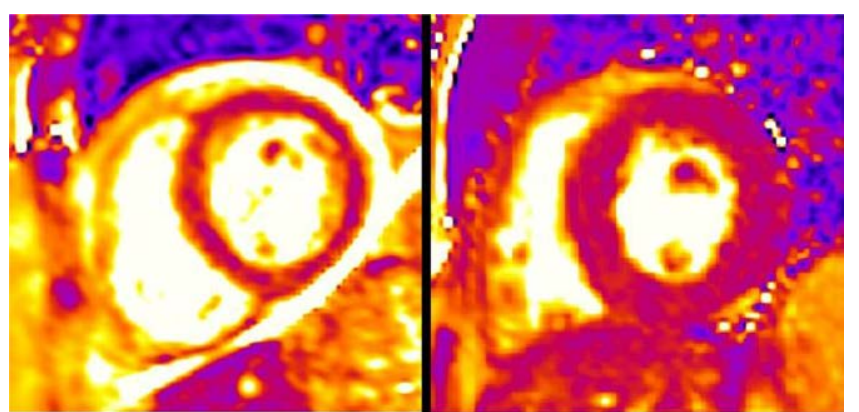

Left panel shows a short-axis $\mathrm{T} 2$ map from Case 1. There is heterogeneity to $\mathrm{T} 2$ mapping values with multiple areas $>55 \mathrm{~ms}$ consistent with myocardial oedema. There is also a pericardial effusion. Right panel shows a short-axis T2 map from Case 2. There is again heterogeneity to T2 mapping with multiple areas $>55 \mathrm{~ms}$ consistent with myocardial oedema. There is also LVH consistent with hypertension.

Conclusion: CMR in acute illness with hyperferritinaemia reveals abnormal tissue characterisation with myocardial inflammation, even when echocardiography is normal. We suggest CMR may be a useful test to expand our understanding of hyperferritinaemic disease states.

\section{References:}

[1] M Gerfaud-Valentin et al. Myocarditis in Adult-Onset Still Disease. Medicine (Baltimore) 2014 Oct; 93(17): 280-289

Disclosure of Interests: None declared

DOI: 10.1136/annrheumdis-2020-eular.2666

\section{$\mathrm{AB} 1024$ \\ EVALUATION OF CLINICAL FEATURES IN PATIENTS DIAGNOSED WITH JUVENILE AND ADULT-ONSET FAMILIAL MEDITERRANEAN FEVER}

D. Erdem Gürsoy ${ }^{1}$, H. H. Gezer², S. Acer Kasman², N. Öz², A. Ozer², M. T. Duruöz ${ }^{2}{ }^{1}$ Adıyaman Training and Research Hospital, Rheumatology, Adıyaman, Turkey; ${ }^{2}$ Marmara University School of Medicine, Department of Physical Medicine and Rehabilitation, Rheumatology Division, Istanbul, Turkey

Background: Familial Mediterranean Fever (FMF), which is more common in groups in the Mediterranean basin, is a monogenic auto inflammatory disease characterized by recurrent attacks of febrile peritonitis, pleuritis and arthritis. Objectives: The aim of this study is to investigate the clinical features of patients diagnosed with juvenile and adult-onset Familial Mediterranean Fever (FMF). Methods: Patients with FMF were included in the study consecutively without sample selection. Data about age, sex, disease duration (month), symptom duration, age at diagnosis, diagnosis delay time, comorbid diseases, and medications were noted. Patients with onset of symptoms $\leq 20$ years old were classified as juvenile-onset, those $>20$ years old were classified as adult-onset FMF. The frequency and characteristics of attacks and the presence of amyloidosis will be recorded

Disease activity was assessed with the PRAS disease activity score. The Health Assessment Questionnaire (HAQ) and SF-36 were used to evaluate physical disability and quality of life, respectively.

The descriptive analysis was done for all parameters. Differences between categorical variables were assessed by Chi-square test and Fisher's exact test. The Mann Whitney-U test was used to compare two sample means. $\mathrm{P}<0.05$ accepted as significant. SPSS 20.0 (Statistical package for social sciences for Windows 20.0) program was used for the statistical analysis.

Results: The mean age of 86 patients ( 63 female, 23 male) with FMF was 38.38 (SD: 12.13 ) years. The patients with the juvenile-onset FMF were $26.7 \%$ of the patients. 